112 (17.0\%), inadequate photo for NICE in 59/112 (52.7\%) and histology processing error in 3/112 (2.7\%).

Conclusions Adequacy of polyp photo is critical to quality assurance of OD. In this study a significant proportion of OD errors had corresponding inadequate photodocumentation with incorrect focal distance the main photographic issue. This may affect application of the NICE criteria. In cases where photos were adequate, understandable error occurred where lesions had a mixture of NICE 1 and 2 features.

\section{PTU-13 LEARNING CURVE OF OPTICAL DIAGNOSIS WITH A RESECT AND DISCARD STRATEGY FOR SCREENING COLONOSCOPY}

Ahmir Ahmad*, Ana Wilson, Siwan Thomas-Gibson, Noriko Suzuki, Adam Humphries, Adam Haycock, Kevin Monahan, Margaret Vance, Angad Dhillon, Brian Saunders. Wolfson Unit for Endoscopy, Imperial College London, London, UK

\subsection{6/gutjnl-2021-BSG.86}

Introduction Advanced endoscopic imaging allows optical diagnosis of diminutive polyps. Performance thresholds for optical diagnosis have been set to allow a 'resect and discard strategy' which may streamline patient care and reduce histopathology need. Aim: to assess early implementation and quality assurance of this strategy in a bowel cancer screening unit setting (1.1 million population).

Methods In this prospective feasibility study, 8 bowel cancer screening colonoscopists completed a validated optical diagnosis training module and performed all procedures during FebOct 2020. All $\leq 5 \mathrm{~mm}$ polyps had white-light and narrow-band images. Endoscopists classified the optical diagnosis as high or low confidence. All polyps had standard histopathology.

Performance was analysed in 2 month time periods with active feedback given. There was repeat histology review of inaccurate high confidence optical diagnoses.

Results In the 8 months to date, 639 polyps were optically diagnosed (468 high confidence and 171 low confidence) from 283 patients who were divided into 4 time periods.

The proportion of low confidence diagnoses increased from $15 \%$ in periods $1-2$ to $29 \%$ in period 4 .

In periods 1-2, 1/8 endoscopists achieved the 90\% accuracy threshold. In periods 3 and $4,2 / 8$ and $4 / 8$ endoscopists achieved the threshold respectively. Overall, accurate diagnoses increased from $81 \%$ to $86 \%$ (period 1 to 4 ). Of those who reached the threshold by period 4 the mean number of high confidence calls was 60 (range 41-99).

For periods 1-4, the positive and negative predictive values for optical diagnosis of an adenoma were $89.3 \%$ and $64.4 \%$ respectively:

\begin{tabular}{lllll} 
Abstract PTU-13 Table 1 & & \\
\hline & & \multicolumn{2}{c}{ Histology } & Total \\
\cline { 2 - 4 } & & Adenoma & $\begin{array}{l}\text { Non- } \\
\text { adenoma }\end{array}$ \\
\hline $\begin{array}{llll}\text { Optical } \\
\text { diagnosis }\end{array}$ & $\begin{array}{l}\text { Adenoma } \\
\text { Non- } \\
\text { adenoma }\end{array}$ & 275 & 57 & 332 \\
Total & 33 & 103 & 136 \\
\hline
\end{tabular}

Conclusions The learning curve for optical diagnosis in a bowel cancer screening setting varies between individual operators. Over time, and with feedback, there is a gradual increase in optical diagnosis accuracy and in the number of colonoscopists achieving the $90 \%$ threshold accuracy target, with a corresponding decrease in the percentage of high confidence calls.

\section{PTU-14 PATIENT ACCEPTABILITY OF DIMINUTIVE POLYP OPTICAL DIAGNOSIS WITH RESECT AND DISCARD STRATEGY IN SCREENING COLONOSCOPY}

Ahmir Ahmad*, Ana Wilson, Siwan Thomas-Gibson, Noriko Suzuki, Adam Humphries, Adam Haycock, Kevin Monahan, Margaret Vance, Angad Dhillon, Brian Saunders. Wolfson Unit for Endoscopy, Imperial College London, London, UK

\subsection{6/gutjnl-2021-BSG.87}

Introduction Colonoscopy with polypectomy reduces the rate of subsequent colorectal cancer and associated mortality. Most polyps resected are diminutive and have a low cancer risk. Currently, these diminutive polyps are resected and examined histologically. Using advanced imaging technologies some studies show we can distinguish adenomas from non-adenomas optically. This could potentially avoid unnecessary polypectomies and associated histology costs in cases where there is high confidence in the diagnosis of the polyps allowing polyps to be resected and discarded. The aim of this study is to assess the acceptability of such a strategy for patients in real life clinical practice.

Methods Two hundred and fifty patients undergoing a bowel cancer screening colonoscopy procedure, where optical diagnosis was used as part of the DISCARD3 study, were invited to participate in a patient experience survey. This was carried out over February - October 2020 at a London bowel cancer screening centre (screening population 1.1 million) with 209 patients included (41 patients excluded: 33 did not complete, 8 did not consent).

Patients were asked to rate the following questions (responses: 5 point scale from strongly disagree to strongly agree):

2. My colonoscopy was comfortable

1. I would be happy for any polyps found during my colonoscopy to be assessed by the person carrying out the procedure at the time (rather than to wait for laboratory results to get the diagnosis)

2. If a future colonoscopy is advised I would prefer to be informed immediately after the initial procedure rather than wait to be told later by telephone or in the outpatient department

3. I feel confident that the person carrying out the procedure could accurately diagnose polyps without sending them to the laboratory

4. I felt highly satisfied with the procedure todayPatients were also asked about their previous experience (responses: Yes, No or N/A):

5. If you have had a previous colonoscopy, did you notice any difference in the procedure today compared with previously?

Results Most patients found their procedure comfortable $(85.2 \% ; 178 / 209)$ and were satisfied with their procedure (97.6\%; 204/209). Most agreed or strongly agreed that they would be happy for polyps to be assessed optically $(78.9 \%$; 